\title{
Nonexponential decay of velocity correlations in surface diffusion: The role of interactions and ordering
}

\author{
Vattulainen, Ilpo Tapio; Hjelt, T.; Ala-Nissila, T.; Ying, S. C.
}

Published in:

Journal of Chemical Physics

Link to article, DOI:

$10.1063 / 1.1322654$

Publication date:

2000

Document Version

Publisher's PDF, also known as Version of record

Link back to DTU Orbit

Citation (APA):

Vattulainen, I. T., Hjelt, T., Ala-Nissila, T., \& Ying, S. C. (2000). Nonexponential decay of velocity correlations in surface diffusion: The role of interactions and ordering. Journal of Chemical Physics, 113(22), 10284-10292. https://doi.org/10.1063/1.1322654

\section{General rights}

Copyright and moral rights for the publications made accessible in the public portal are retained by the authors and/or other copyright owners and it is a condition of accessing publications that users recognise and abide by the legal requirements associated with these rights.

- Users may download and print one copy of any publication from the public portal for the purpose of private study or research.

- You may not further distribute the material or use it for any profit-making activity or commercial gain

- You may freely distribute the URL identifying the publication in the public portal 


\title{
Nonexponential decay of velocity correlations in surface diffusion: The role of interactions and ordering
}

\author{
I. Vattulainen \\ Department of Chemistry, Technical University of Denmark, Building 207, DK-2800 Lyngby, Denmark \\ T. Hjelt \\ Helsinki Institute of Physics and Laboratory of Physics, Helsinki University of Technology, P.O. Box 1100, \\ FIN-02015 HUT, Espoo, Finland \\ T. Ala-Nissila \\ Helsinki Institute of Physics and Laboratory of Physics, Helsinki University of Technology, P.O. Box 1100, \\ FIN-02015 HUT, Espoo, Finland and Department of Physics, Brown University, Providence, \\ Rhode Island 02912-1843
}

S. C. Ying

Department of Physics, Brown University, Providence, Rhode Island 02912-1843

(Received 27 June 2000; accepted 13 September 2000)

\begin{abstract}
We study the diffusive dynamics of adparticles in two model systems with strong interactions by considering the decay of the single-particle velocity correlation function $\phi(t)$. In accordance with previous studies, we find $\phi(t)$ to decay nonexponentially and follow a power-law $\phi(t) \sim t^{-x}$ at intermediate times $t$, while at long times there is a crossover to an exponential decay. We characterize the behavior of the decay exponent $x$ in detail in various ordered phases and in the vicinity of phase boundaries. We find that within the disordered phase, the behavior of $x$ can be rationalized in terms of interaction effects. Namely, $x$ is typically larger than two in cases where repulsive adparticle-adparticle interactions dominate, while attractive interactions lead to $x<2$. In ordered phases, our results suggest that the behavior of $x$ is mainly governed by ordering effects that determine the local structure in which adatoms diffuse. Then the decay is characterized by $1<x$ $<2$ under conditions where diffusion is truly two-dimensional, while in phases where adatoms diffuse in a one-dimensional fashion along ideal rows of vacancies, we find a regime characterized by $x<1$. Also, changes in the qualitative behavior of $x$ are closely related to phase boundaries and local ordering effects. Our studies suggest that $\phi(t)$ can be used to obtain information about the ordering of the system and about the nature of predominant interactions between adparticles. Our predictions can be tested experimentally by techniques such as scanning tunneling microscopy, in which $\phi(t)$ can be measured in terms of discrete adparticle displacements as shown in this work. Finally, our studies suggest that the decay of velocity correlations in collective diffusion follows, qualitatively, the same behavior as the decay of single-particle velocity correlations in tracer diffusion. (C) 2000 American Institute of Physics. [S0021-9606(00)70246-7]
\end{abstract}

\section{INTRODUCTION}

In various technologically important processes such as surface growth ${ }^{1}$ and chemical reactions, ${ }^{2}$ the dynamics of individual tagged particles diffusing on surfaces plays a fundamental role. The quantity that characterizes the motion of such particles is the tracer diffusion coefficient ${ }^{3}$

$$
D_{T}=\lim _{t \rightarrow \infty} \frac{1}{2 d t}\left\langle|\mathbf{r}(t)-\mathbf{r}(0)|^{2}\right\rangle,
$$

where $d=2$ is the dimensionality of the system and $\mathbf{r}(t)$ is the position of the tagged particle at time $t$. Equivalently, $D_{T}$ can be defined in terms of the velocity autocorrelation function $\phi(t) \equiv\langle\mathbf{v}(t) \cdot \mathbf{v}(0)\rangle$ through the Kubo-Green formula, ${ }^{4}$

$$
D_{T}=\frac{1}{d} \int_{0}^{\infty} \phi(t) d t
$$

where $\mathbf{v}(t)$ represents the velocity of the tagged particle. Both approaches are commonly used to calculate the tracer diffusion coefficient $D_{T}$ and therefore to characterize the motion of individual tagged particles at long time and length scales in the hydrodynamic limit. However, it is obvious from Eq. (2) that the most significant contribution to $D_{T}$ arises from short-time correlations in the motion of the tagged particle, correlations that are quantified by the timedependence of $\phi(t)$. The velocity correlation function $\phi(t)$ is thus the central quantity that provides useful information of the dynamics of a given tagged particle.

Another quantity often used in describing surface diffusion is the collective (chemical) diffusion coefficient $D_{C}$, which describes the decay rate of collective density fluctuations in equilibrium and is closely related to various technological applications such as lubrication and mass transfer during relaxation of surface systems after growth. $D_{C}$ can be conveniently expressed via the Kubo-Green formula ${ }^{4}$ 


$$
D_{C}=\frac{1}{k_{B} T \theta \kappa_{T} N d} \int_{0}^{\infty} \psi(t) d t
$$

where $\theta$ is the coverage of the particles that form an adlayer on the surface, $N$ is the total number of particles, and $\kappa_{T}$ is the compressibility which is related to particle number fluctuations within a probe area. The main quantity we wish to focus on in Eq. (3) is the flux correlation function $\psi(t)$ $\equiv\langle\mathbf{J}(t) \cdot \mathbf{J}(0)\rangle$, where $\mathbf{J}(t)=\sum_{i=1}^{N} \mathbf{v}_{i}(t)$ is the flux of all diffusing adparticles $i=1, \ldots, N$ in terms of their velocities $\mathbf{v}_{i}(t)$. It plays a role similar to the single-particle velocity correlation function $\phi(t)$ in that the prefactor in Eq. (3) contains no dynamic information but the dynamics is wholly described by the flux correlation function $\psi(t)$. It is important to note that for interacting systems at higher coverages, $D_{C}$ can be very different from $D_{T}$ since $D_{C}$ is only somewhat indirectly related to the motion of individual tagged particles. ${ }^{3,5}$

Previous surface diffusion studies of the motion of adatoms and more complex molecules have mainly focused on how the surface diffusion coefficients $D_{T}$ and $D_{C}$ depend on thermodynamic conditions such as the presence of various ordered phases and phase boundaries. ${ }^{3,5-7}$ Also various studies have revealed intriguing insight of the microscopic diffusion mechanisms by which adparticles migrate along the surface. ${ }^{8-12}$ Thus there is considerable knowledge of the long-time limit characterized by the diffusion coefficients, and also of the various microscopic mechanisms by which adparticles move along the surface. However, much less is known about the dynamics at short and intermediate times, as described by the correlation functions $\phi(t)$ and $\psi(t)$.

In surface systems, the dynamics of adparticle motion arises first from intrawell motion within an adsorption site and then from interwell motion due to migration from one adsorption site to another. If the average transition rate for single-particle jumps is denoted by $\Gamma$, then the fundamental time unit of mass transport along the plane is the inverse escape time $\tau_{e}=1 / \Gamma$. For times $t \gtrsim \tau_{e}$, where vibrational motion is no longer accounted for, it is well known that lattice-gas descriptions of surface systems work reasonably well at low temperatures. ${ }^{6}$ Then it has been shown that the leading terms of the correlation functions $\phi(t)$ and $\psi(t)$ are proportional to $\Gamma \cdot{ }^{13,14}$ The understanding of the behavior of $\phi(t)$ and $\psi(t)$ beyond this regime is much more limited, and constitutes a fundamental problem since they must decay rapidly enough to give finite diffusion coefficients according to Eqs. (2) and (3). Some knowledge is provided by the understanding of the motion of a Brownian particle whose mass is much larger than those of the surrounding solvent particles, in which case $\phi(t)$ decays exponentially in time reflecting the lack of memory effects. ${ }^{4}$ In surface diffusion studies an exponential decay of $\phi(t)$ is observed, if adatom diffusion is considered to be the Brownian motion of independent particles in a periodic potential, with uncorrelated collisions with the substrate excitations. ${ }^{5,15,16}$

In real systems of interacting particles, the behavior of $\phi(t)$ and $\psi(t)$ is much more complicated. ${ }^{15,16}$ Even in the case of individual adatoms, the decay of $\phi(t)$ depends on the adatom-substrate coupling and may show behavior very different from pure exponential decay. ${ }^{17}$ Another well-known example is self-diffusion in a fluid, in which the conservation of momentum drives the particles to diffuse in a concerted manner under long-ranged hydrodynamic interactions, thus leading to an algebraic decay $\phi(t) \sim t^{-x}$ with an exponent $x=d / 2$. $^{4,18,19}$ In two-dimensional (2D) adsorption systems, however, the situation is very different. The diffusion processes are driven by thermal fluctuations, and dissipation occurs both through a frictional coupling to the substrate and through interparticle interactions. The absence of hydrodynamic interactions and related conservation laws is therefore evident, and thus a long-time power-law decay of $\phi(t)$ is not to be expected.

However, recent Monte Carlo and molecular dynamics studies of some 2D adsorption systems ${ }^{14,20}$ and dissipative 2D hard sphere fluids ${ }^{21}$ have revealed an intermediate algebraic decay of velocity correlations in these systems, spanning times extending up to about two orders of magnitude in the case of strong interactions. ${ }^{14,20}$ Results of similar nature have been found for the decay of $\psi(t)$ in 2D adsorption systems. ${ }^{14}$ What is possibly most exciting is that recent studies of $\phi(t)$ suggest its power-law decay at intermediate times to be a generic feature in interacting dissipative 2D systems,${ }^{20}$ since the decay has been observed in the Langmuir gas model in which the only interaction between adparticles is to exclude double-occupancy of the adsorption sites, as well as in various interacting surface systems. This provides strong evidence for the idea that the intermediate power-law decay is in part of entropic origin. The decay exponent is not universal, however, since $x$ has been shown to depend on the range and the strength of adparticleadparticle interactions. ${ }^{20}$ How this occurs is not known at present. Besides of fundamental theoretical interest, it would be of practical use to be able to correlate the value of $x$ as extracted from measurements ${ }^{3,22}$ of $\phi(t)$ or $\psi(t)$ with, e.g., interactions and spatial ordering in a given adsorbate system.

In this work, our purpose is to clarify this issue through studies of $\phi(t)$ and $\psi(t)$ in two strongly interacting $2 \mathrm{D}$ model systems via Monte Carlo (MC) simulations. In most cases studied, we indeed find a nonexponential regime at intermediate times where the decay can be well approximated by power laws such as $\phi(t) \propto t^{-x}$ and $\psi(t) \propto t^{-y}$. We then focus on the behavior of $x$ and characterize its behavior in detail in various ordered phases and in the vicinity of phase boundaries. We find that in a disordered phase the behavior of $x$ can reasonably well be explained in terms of interparticle interactions. Namely, we find that $x$ is typically larger than two in cases where repulsive adparticleadparticle interactions dominate, while attractive interactions lead to $x<2$. In ordered phases, we find that predominantly repulsive interactions lead to $x$ that is larger than the exponent obtained from predominantly attractive interactions. However, in addition we find that within ordered phases $x$ depends very strongly on the degree of order in the system and has complex behavior depending on the phase in which adatoms diffuse. The decay is characterized by $1<x<2$ under conditions where diffusion is truly two-dimensional, while almost one-dimensional diffusion along ideal rows of vacancies in an ordered $p(2 \times 1)$ phase leads to $x<1$. Furthermore we find that changes in $x$ are related to phase 
boundaries and to changes in ordering within the ordered phases. Additional studies of $\psi(t)$ suggest that the decay of velocity correlations in collective diffusion is qualitatively similar to the decay of $\phi(t)$, although the decay of $\psi(t)$ is much more rapid in most cases. Our results propose that $\phi(t)$ and $\psi(t)$ can be used to obtain information about the ordering of the system and about the nature of predominant interactions between adparticles. The predictions presented here can be tested experimentally by techniques such as scanning tunneling microscopy (STM), ${ }^{22}$ in which velocity correlations can be measured in terms of discrete adparticle displacements as shown in this work.

\section{VELOCITY CORRELATION FUNCTIONS}

In continuum simulation methods such as molecular dynamics and Brownian dynamics, velocity correlation functions are readily available. In experiments and in coarsegrained simulation methods such as lattice Monte Carlo, however, one only observes particle positions at discrete times, and thus extracting information of velocity correlations in these cases is not obvious. Recently, the so-called "memory expansion" method (MEM) has been introduced $^{14,20}$ which can be used to obtain information of velocity correlations from particle displacement data. This method provides an efficient way to compute the tracer and the collective diffusion coefficients $D_{T}$ and $D_{C}$ in general, too. Here we briefly outline this approach by presenting the memory expansions of tracer and collective diffusion in 2D and then relating them to velocity correlations.

\section{A. Tracer diffusion}

Within the MEM for tracer diffusion, one divides the time $t$ into $M$ time intervals of equal length $\tau_{0}$, and discretizes the positions correspondingly at times $t_{m}=m \tau_{0}$ with $m=0,1, \ldots, M$. Then $\mathbf{r}(t) \equiv \mathbf{r}\left(M \tau_{0}\right)=\sum_{m=1}^{M} \delta \mathbf{R}\left(t_{m}\right)$, where $\delta \mathbf{R}\left(t_{m}\right)=\mathbf{r}\left(t_{m}\right)-\mathbf{r}\left(t_{m-1}\right)$ is the change in the position of the tagged particle between two consecutive observations at times $t_{m}$ and $t_{m-1}$. Substitution of this result into Eq. (1) and exploiting the time translational invariance leads to the expression $^{14}$

$$
D_{T}=\frac{\tau_{0}}{4}\left[\phi_{g}(0)+2 \sum_{k=1}^{\infty} \phi_{g}\left(k \tau_{0}\right)\right]
$$

in terms of the generalized velocity autocorrelation functions

$$
\phi_{g}(t)=\left\langle\mathbf{v}_{g}\left(t_{m}+t\right) \cdot \mathbf{v}_{g}\left(t_{m}\right)\right\rangle,
$$

where we have defined the generalized velocity $\mathbf{v}_{g}\left(t_{m}\right)$ $\equiv \delta \mathbf{R}\left(t_{m}\right) / \tau_{0}$. Obviously, in the limit where the time step $\tau_{0} \rightarrow 0$, this reduces to the traditional definition of $\phi(t)$ for the continuous case and the summation formula in Eq. (4) reduces to the familiar Kubo-Green formula in Eq. (2).

We note that in the limit of small time displacements $\tau_{0}$, even the leading term $\phi_{g}(0)$ in Eq. (4) provides useful information of adatom motion, since in the low-temperature limit for a lattice-gas model $\phi_{g}(0)=a^{2} \Gamma / \tau_{0}$, where $a$ is the jump length and $\Gamma$ the average transition rate of singleparticle jumps. Then the remaining terms $\phi_{g}(t>0)$ characterize dynamical correlations (memory effects) between con- secutive displacements of a given particle, and give rise to the correlation factor $f_{T}$. When combined, one finds that $D_{T}$ can be described by $D_{T}=a^{2} \Gamma f_{T} / 4{ }^{6,13,23}$ This example demonstrates how studies of short-time velocity correlations can be related to the long-time diffusive behavior.

\section{B. Collective diffusion}

For collective diffusion, we proceed in a manner very similar to tracer diffusion. Thus we consider particles $i$ $=1, \ldots, N$ whose positions are denoted by $\mathbf{r}_{i}(t)$. We then divide the time $t$ into $M$ time intervals of equal length $\tau_{0}$ and discretize the position of the center-of-mass $\mathbf{R}_{\mathrm{cm}}(t)$ $=\sum_{i=1}^{N}\left[\mathbf{r}_{i}(t)-\mathbf{r}_{i}(0)\right]$ at times $t_{m}=m \tau_{0}$ with $m=0,1, \ldots, M$. Then $\quad \mathbf{R}_{\mathrm{cm}}(t) \equiv \mathbf{R}_{\mathrm{cm}}\left(M \tau_{0}\right)=\Sigma_{m=1}^{N} \delta \mathbf{R}_{\mathrm{cm}}\left(t_{m}\right), \quad$ where $\delta \mathbf{R}_{\mathrm{cm}}\left(t_{m}\right)=\mathbf{R}_{\mathrm{cm}}\left(t_{m}\right)-\mathbf{R}_{\mathrm{cm}}\left(t_{m-1}\right)$ is the change in the position of the center-of-mass between two consecutive observations at times $t_{m}$ and $t_{m-1}$. This approach allows us to write the collective diffusion coefficient as ${ }^{14}$

$$
D_{C}=\frac{1}{k_{B} T \theta \kappa_{T}} \frac{\tau_{0}}{4 N}\left[\psi_{g}(0)+2 \sum_{k=1}^{\infty} \psi_{g}\left(k \tau_{0}\right)\right]
$$

in terms of the generalized flux correlation functions

$$
\psi_{g}(t)=\left\langle\mathbf{J}_{g}\left(t_{m}+t\right) \cdot \mathbf{J}_{g}\left(t_{m}\right)\right\rangle,
$$

where we have defined the generalized flux $\mathbf{J}_{g}\left(t_{m}\right)$ $\equiv \delta \mathbf{R}_{\mathrm{cm}}\left(t_{m}\right) / \tau_{0}$. Again it is obvious that in the limit where $\tau_{0} \rightarrow 0$, this reduces to the familiar Kubo-Green formula in Eq. (3) for a continuous case. ${ }^{14}$ Also it is worthwhile pointing out that in the limit of small time steps $\tau_{0}$ for systems that can be described by a lattice-gas description, the leading term $\psi_{g}(0)=a^{2} \Gamma N / \tau_{0} \cdot{ }^{14}$ Therefore $\psi_{g}(0) \propto \Gamma$ in analogy to the case of tracer diffusion. The remaining terms $\psi_{g}(t)$ for $t>0$ characterize dynamical correlations between consecutive displacements of the center-of-mass of the adlayer.

\section{Time scales}

In practice, the choice of the time step $\tau_{0}$ is not trivial and requires some discussion. First, we wish to emphasize that the memory expansions for $D_{T}\left[\mathrm{Eq}\right.$. (4)] and $D_{C}[\mathrm{Eq}$. (6)] are formally exact for any $\tau_{0}$. Yet its practical choice is dictated by the minimization of computational cost. Very small values of $\tau_{0}$ provide an overwhelming amount of information of particle displacements, which is not necessary in calculating the diffusion coefficients. On the other hand, very large values of $\tau_{0}$ are also not very efficient. For calculation of $D_{T}$ and $D_{C}$, it is in most cases best to use $\tau_{0}$ which is larger than a typical microscopic time scale, but less than the time it takes for the correlation functions to decay away. ${ }^{14}$ The situation is slightly different when one aims to calculate the generalized velocity and flux correlation functions $\phi_{g}(t)$ and $\psi_{g}(t)$. In principle, in this case $\tau_{0}$ should be as small as possible to provide the best possible approximation of the true correlation functions $\phi(t)$ and $\psi(t)$. In practice, however, we have found that time steps of reasonably large size [of the order of 10 Monte Carlo steps (MCS's) or more $\left.{ }^{20}\right]$ lead to $\phi_{g}(t)$ which is essentially identical with $\phi(t)$. This can be verified by calculating the incoherent scattering function whose memory function yields $\phi(t) .{ }^{4,24}$ In 
principle, a similar comparison can be made between $\psi_{g}(t)$ and the coherent scattering function whose memory function yields $\psi(t) .{ }^{4,24}$ In practice, however, this comparison is very difficult to make due to noise that renders the calculation of memory functions within the Laplace description impracti$\mathrm{cal}$, and has therefore not been done in the present work.

\section{Analysis of power-law behavior}

It is well known that extracting exponents from the power-law type of behavior of correlation functions is a difficult task due to e.g. crossover effects and noise. In this work, we have addressed this problem by analyzing the power-law behavior of the velocity and the flux correlation functions by two different means. We have first considered $\phi_{g}(t)$ vs time $t$ on a $\log -\log$ scale, and extracted the exponent $x$ from a regime where its instantaneous value (over a narrow time window) fluctuates around a constant level. This approach was used in our recent studies where $\phi_{g}(t)$ was demonstrated to decay like a power law. ${ }^{14}$ Second, we have made use of the fact that $\phi_{g}(t=0)$ is a finite but usually large factor with regard to other terms at times $t>0$. If this is the case, then the large leading term may prevent a reliable determination of $x$ at intermediate times. To consider this possibility, we have described $\phi_{g}(t)$ by ${ }^{25}$

$$
\phi_{g}(t)=\frac{\phi_{g}(0)}{1+A t^{x}},
$$

which leads to an asymptotic decay $\phi_{g}(t) \sim t^{-x}$ for $A t^{x} \gg 1$. In a similar fashion, we used the two approaches to analyze the power-law behavior of $\psi_{g}(t)$.

In the present work, we have found that the two approaches suggested above are mutually consistent and give results for $x$ and $y$ that are equal within the error bars. However, in some cases we have found that the second approach detects the initial power-law behavior better than the first one. For such situations, we define the correlation functions

$$
\widetilde{\phi}_{g}(t) \equiv\left[\frac{\phi_{g}(t)}{\phi_{g}(0)}\right]^{-1}-1
$$

and

$$
\widetilde{\psi}_{g}(t) \equiv\left[\frac{\psi_{g}(t)}{\psi_{g}(0)}\right]^{-1}-1,
$$

whose results will be presented below when appropriate. Evidently these correlation functions are expected to behave as $\widetilde{\phi}_{g}(t) \sim t^{x}$ and $\widetilde{\psi}_{g}(t) \sim t^{y}$ at intermediate times.

\section{MODEL SYSTEMS}

\section{A. Adsorption system O/W(110)}

We first study the diffusion of oxygen adatoms on a W(110) surface in a lattice-gas model with Monte Carlo dynamics. The oxygen-oxygen interaction Hamiltonian contains pair interactions and also a contribution from threebody interactions that are important at large coverages. These lead to the appearance of a number of ordered phases and coexistence regions such that the resulting phase diagram shown in Fig. 1 is in close agreement with the experi-

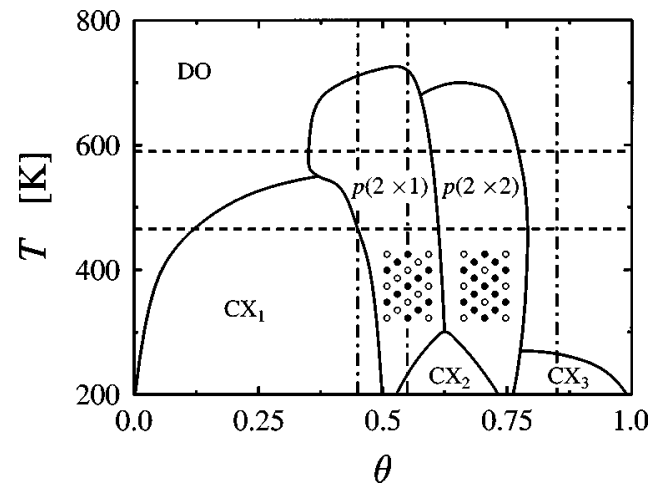

FIG. 1. A schematic phase diagram of the $\mathrm{O} / \mathrm{W}(110)$ system in the $T-\theta$ plane. DO denotes the disordered region, $p(2 \times 1)$ and $p(2 \times 2)$ denote the ordered phases, and $C X_{i}$ with $i=1,2,3$ are coexistence phases. Snapshots of ideal ordered phases are also shown, the occupied and vacant adsorption sites denoted by filled and open circles, respectively. The coverage regimes 1(a) -1 (c) and the temperature regimes 2(a)-2(b) (see Sec. III A) studied in this work are shown with dashed-dotted and dashed lines, respectively.

mental observations ${ }^{26-28}$ for the O/W(110) system. Details of this model system and the Hamiltonian parameters can be found elsewhere. ${ }^{23,29}$

We wish to stress that in Monte Carlo studies of dynamical properties such as velocity correlations and diffusion coefficients, the specification of the interaction parameters in the lattice-gas Hamiltonian is not alone sufficient. The results also depend on the choice of transition probabilities which determine the dynamics in a given system. ${ }^{30}$ In the present work, we have used the so-called Transition Dynamics Algorithm which models diffusion as a thermally activated process over a diffusion barrier, whose height depends on the adatom-adatom interactions as well as the adiabatic substrate potential. Further details and additional references of this approach can be found in Refs. 29 and 30, where we have introduced this method and justified its use by various comparisons to other dynamical algorithms and models studied via molecular dynamics simulations.

In the present study we will first concentrate on the temperature dependence of velocity correlations as one comes from the high-temperature disordered phase down to various ordered phases at lower temperatures. To this end, we consider three different cases (see Fig. 1):

1(a) The first case is at a coverage of $\theta=0.45$, where there is a continuous phase transition from the disordered phase at $T>710 \mathrm{~K}$ to an ordered $p(2 \times 1)$ phase between $420 \mathrm{~K}<T<710 \mathrm{~K}$. At lower temperatures, there is a coexistence of the $p(2 \times 1)$ and DO phases through a first-order boundary at about $420 \mathrm{~K}$.

1(b) At a slightly higher coverage of $\theta=0.55$, we start from the DO phase at $T>720 \mathrm{~K}$ and then go continuously down to the $p(2 \times 1)$ phase at lower temperatures. At very low temperatures $(T<250 \mathrm{~K})$, there is yet another coexistence phase.

1(c) Eventually, we consider the high-coverage case at $\theta=0.85$ which is characterized by the DO phase at temperatures $T>270 \mathrm{~K}$ and a coexistence phase below this temperature.

Second, we additionally consider the coverage depen- 


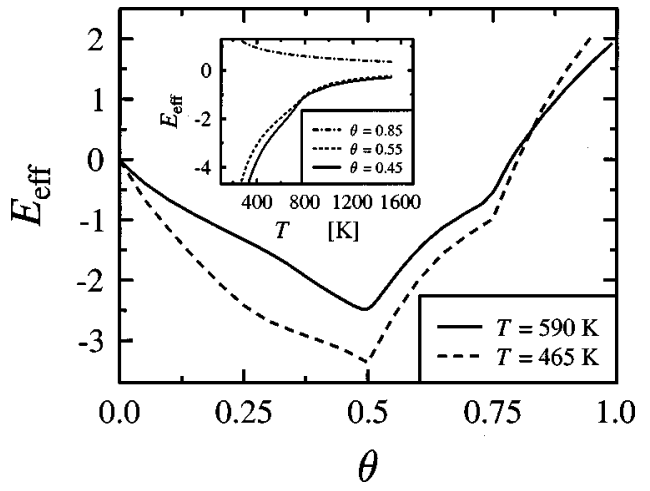

FIG. 2. Results for the average energy per adatom with respect to the corresponding thermal energy, $E_{\text {eff }} \equiv\langle\mathcal{H}\rangle / k_{B} T N$ where $\mathcal{H}$ is the Hamiltonian of the direct adatom-adatom interactions in the $\mathrm{O} / \mathrm{W}(110)$ model system (Ref. 29). The results for the coverage dependence of $E$ are shown in the main figure, while the temperature dependence is illustrated in the inset.

dence of the correlation functions at two different temperature regions at reasonably low temperatures. These regions are characterized by the following features (see again Fig. 1):

2(a) The first temperature region at $590 \mathrm{~K}$ is characterized by a disordered phase (DO) at low coverages, from which it crosses over to an ordered $p(2 \times 1)$ phase at $\theta$ $=0.35$. At higher coverages, there is another transition to an ordered $p(2 \times 2)$ phase at $\theta=0.59$, which in turn crosses over to a disordered phase at $\theta=0.78$. All these transitions are continuous.

2(b) In the second region around $465 \mathrm{~K}$, there is again a disordered phase at very low coverages. At $0.12 \leqslant \theta \leqslant 0.45$, there is a coexistence phase of the DO and the $p(2 \times 1)$ phases bounded by first order phase transition boundaries. The $p(2 \times 1)$ phase around $\theta=0.5$ then crosses continuously over to the $p(2 \times 2)$ phase at $\theta=0.63$, which in turn crosses continuously over to the disordered phase at $\theta=0.81$.

Finally, for the purpose of forthcoming discussion, we show in Fig. 2 results for the effective average energy per adatom

$$
E_{\mathrm{eff}} \equiv \frac{1}{N} \frac{\langle\mathcal{H}\rangle}{k_{B} T},
$$

where $\mathcal{H}$ is the Hamiltonian of the present model system. ${ }^{29}$ We may conclude that attractive adatom-adatom interactions dominate at small coverages, while at large covarages the behavior of the system is governed by repulsive interactions.

\section{B. Model polymer system}

Additionally, we study a model of flexible, chainlike molecules on flat surfaces. The chains are modeled by the 2D fluctuating-bond model with Monte Carlo dynamics, ${ }^{31,32}$ in which each segment excludes four nearest and next-nearest neighbor sites on a square lattice. The exclusion induces a strong entropic repulsion between the molecules even if there are no direct interactions present. The repulsion strongly influences diffusion in this system, and has been studied in detail in Ref. 33. In this work, we concentrate on the case where there is an additional direct Lennard-Jones

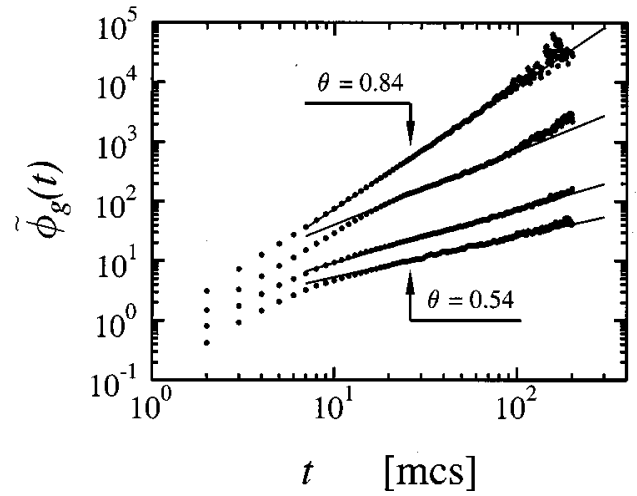

FIG. 3. Typical results for the decay of $\widetilde{\phi}_{g}(t)$ at $T=465 \mathrm{~K}$ in the O/W(110) model system up to times, where the noise becomes too pronounced or where the decay starts to cross over to an exponential form. Results are shown for four coverages from top to bottom: $\theta=0.84,0.74,0.59$, and 0.54 . Full lines are fits to the data. Some curves have been shifted for clarity.

(LJ) type of attraction between the segments of different chains. This model allows a systematic tuning of the interplay between repulsive and attractive interactions. Details on the model and parameters can be found in Ref. 14.

\section{RESULTS FOR VELOCITY CORRELATIONS IN TRACER DIFFUSION}

\section{A. Results for the model system O/W(110)}

To determine the decay of the generalized velocity correlation function, we calculated $\phi_{g}(t)$ with a time step of $\tau_{0}=1$ MCS over a wide temperature and coverage range as explained in Sec. III A. The correlation function $\phi_{g}(t)$ has a positive leading term $\phi_{g}(0)$ and a negative tail at times $t$ $>0$, where the tail arises from backcorrelation effects that are typical for tracer diffusion. ${ }^{23}$ Thus we simplify the analysis by omitting the leading term and only consider the tail $\phi_{g}(t>0)$. Equation (9) then comes into the form $\widetilde{\phi}_{g}(t)$ $=\left[\phi_{g}(t+1) / \phi_{g}(1)\right]^{-1}-1$, where the time $t$ is now given in units of one MCS per particle. Typical results for $\widetilde{\phi}_{g}(t)$ are shown in Fig. 3. We find that after some small crossover time, a power-law form $\widetilde{\phi}_{g}(t) \propto t^{x}$ develops at intermediate times, and is the essence of the following discussion.

\section{Temperature dependence}

In all cases, we have determined the effective exponent $x$ from the intermediate time regime in which the power-law form $\widetilde{\phi}_{g}(t) \propto t^{x}$ (as well as the decay $\phi_{g}(t) \propto t^{-x}$ ) holds true to a good degree of approximation. Results for $x$ as a function of temperature at $\theta=0.45, \theta=0.55$, and $\theta=0.85$ are shown in Fig. 4. Additionally there are some configuration snapshots in Fig. 5 to illustrate typical superstructures in which adatoms diffuse.

First, let us focus on the high-temperature limit which corresponds to the well-known Langmuir gas model, in which adatoms interact only via a hard-core interaction to forbid double occupancy of lattice sites. In this limit it can be shown from an analytical solution of $\phi(t)$ that there is a power-law type of intermediate decay with an exponent $x$ $\approx 2$ for large coverages. ${ }^{20,34,35}$ The exponent $x \approx 2$ therefore 


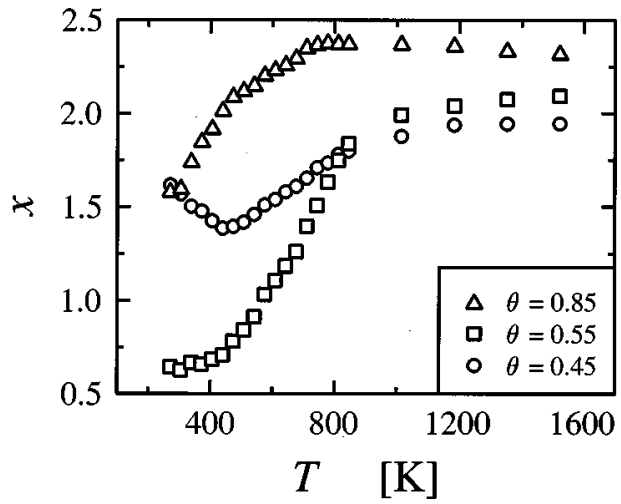

FIG. 4. Results for the temperature dependence of the fitted decay exponent $x$ at coverages $\theta=0.45, \theta=0.55$, and $\theta=0.85$ in the $\mathrm{O} / \mathrm{W}(110)$ model system. Error bars are smaller than the size of the symbols.

characterizes adatom diffusion within a totally disordered phase. In the present case for the $\mathrm{O} / \mathrm{W}(110)$ model, we find that $x \rightarrow 2$ at high temperatures as shown in Fig. 4.

At lower temperatures, the effects of direct adatomadatom interactions and ordering come into play and strongly influence the behavior of $x$. This is clearly demonstrated in Fig. 4. At $\theta=0.45$, the decay exponent $x$ decreases monotonously as the temperature is decreased to about 460 $\mathrm{K}$, after which a further decrease of $T$ leads to an increase of $x$. It is worthwhile pointing out that there is no drastic change in the behavior of $x$ around the continuous phase boundary at $T=710 \mathrm{~K}$, whereas a dip in $x$ seems to coincide with the first-order phase boundary between the $p(2 \times 1)$ phase and the coexistence between the DO and the $p(2 \times 1)$ phases. One way to analyze this behavior is to consider interaction effects. As is evident from Fig. 2, the phase behavior at $\theta$ $=0.45$ is dominated by attractive interactions whose role becomes more important with decreasing temperatures. The attractive interactions lead to a slower decay of velocity cor-

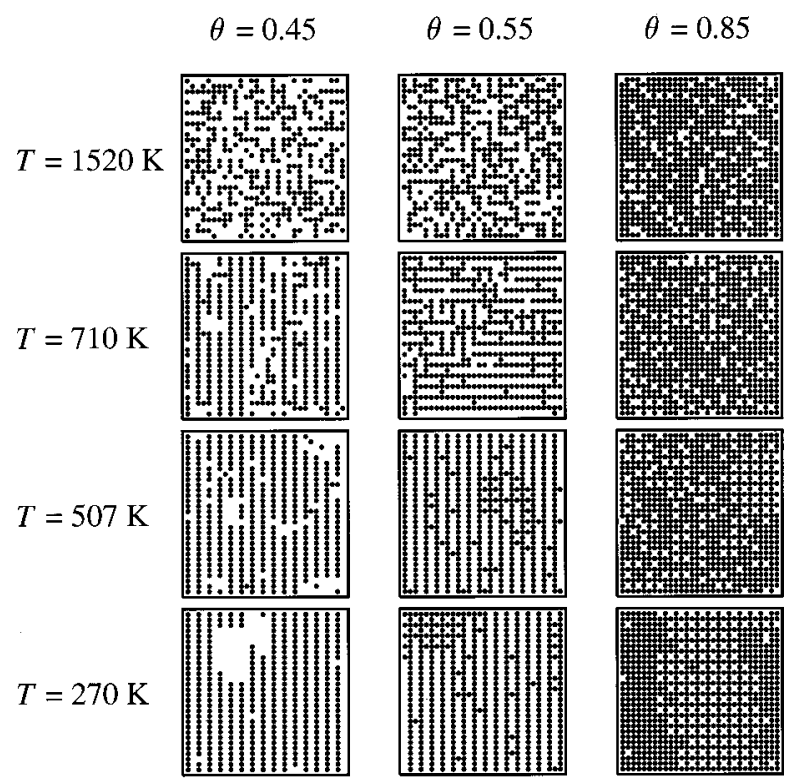

FIG. 5. Configuration snapshots at three different coverages and four temperatures. Occupied adsorption sites are denoted by filled circles. relations with respect to the Langmuir gas limit, and are manifested as a decreasing exponent $x<2$. This suggests that the strength of effective interactions is the main factor that dominates the behavior of $x$. However, this is not entirely true, since at $T=420 \mathrm{~K}$ the correlation between $E$ and $x$ breaks up. This is the first indication of the role played by ordering effects that give rise to peculiar correlations between consecutive displacements of the tagged particle and therefore also affect the behavior of $x$. Indeed, at $T=420 \mathrm{~K}$ one enters the coexistence phase in which diffusion proceeds mainly by the motion of adparticles within the DO phase. Therefore, although the effective adatom-adatom interactions tend to be more and more attractive as the temperature is lowered, the decay exponent tends to increase towards $x$ $\approx 2$ due to the DO phase within the coexistence region.

The situation at $\theta=0.55$ differs very clearly from the previous case in two respects. First, at temperatures $1000 \mathrm{~K}$ $<T<1400 \mathrm{~K}$ in the disordered phase we find $x \geqslant 2$. This behavior is characteristic for systems that are dominated by repulsive interactions ${ }^{20}$ beyond those within the hard-core repulsion. In the present case, we find from Fig. 2 that the role of repulsive interactions builds up at $\theta>0.50$, and is probably the reason for the exponent $x \geqslant 2$ observed. Thus there seems to be a crossover from the high-temperature behavior $(x>2)$ in the DO phase to a low-temperature behavior $(x<2)$ in the ordered $p(2 \times 1)$ phase. Another major difference concerns the low-temperature behavior at $T$ $<580 \mathrm{~K}$. In this region, we find a crossover from 2D adatom diffusion above $580 \mathrm{~K}$ to effectively $1 \mathrm{D}$ diffusion below this crossover temperature. This drastic change in the diffusion behavior is clearly demonstrated by the snapshots in Fig. 5 . This finding is also in agreement with previous studies, in which we observed that right above $\theta=0.50$ at $590 \mathrm{~K}$, there are just a few adatoms that perform effectively onedimensional diffusion along the channels in the $p(2 \times 1)$ phase. $^{23}$ The crossover from $2 \mathrm{D}$ to $1 \mathrm{D}$ diffusion is furthermore very clear, and is supported by recent studies of directional correlations under similar conditions. ${ }^{23}$ Therefore since we do observe a power-law decay of $\phi_{g}(t)$ but with a small exponent $0.6<x<1$, we conclude that exponents $x$ $<1$ are typical for situations in which adatom diffusion is effectively one-dimensional. In the present case, we furthermore expect that decreasing $T$ below $250 \mathrm{~K}$ would lead to an increase of $x$, since then one would enter the coexistence region between the $p(2 \times 1)$ and $p(2 \times 2)$ phases, in which diffusion is at least in part two-dimensional in character.

The last example at $\theta=0.85$ is in agreement with previous conclusions. We first find $x \approx 2$ at very high temperatures in the disordered phase. Then lowering the temperature to about $800 \mathrm{~K}$ leads to an increase of $x$ up to about 2.4 as a result of repulsive interactions, whose role becomes more pronounced at lower temperatures as is illustrated in the inset of Fig. 2. Then, a further decrease of $T$ leads to a decrease of $x$ as the system develops short-range order in the vicinity of the phase boundary between the $p(2 \times 2)$ and DO phases (see snapshots in Fig. 5). This is just another sign of ordering effects, which again are competing with predominant interaction effects. 


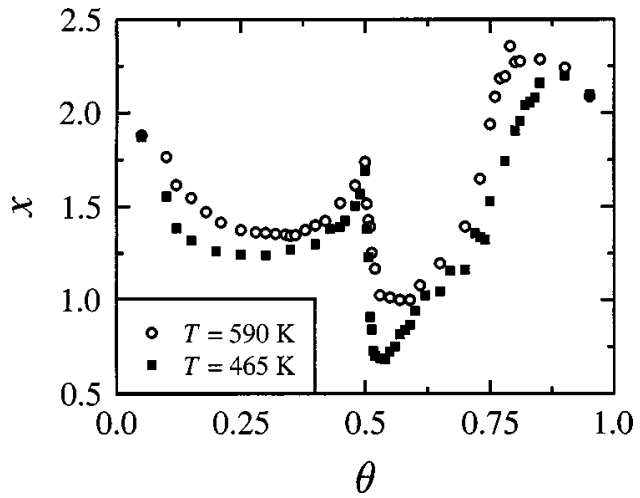

FIG. 6. Results for the coverage dependence of the decay exponent $x$ at temperature $T=465 \mathrm{~K}$ and $T=590 \mathrm{~K}$. Error bars are approximately of the size of the symbols.

\section{Coverage dependence}

We next consider the coverage dependence of the decay exponent $x$ at $T=590 \mathrm{~K}$ and $T=465 \mathrm{~K}$ (see the phase diagram in Fig. 1). As is evident from Fig. 6, in both cases the coverage dependence of $x$ is very similar. First the exponent $x$ decreases at small coverages, then increases from $\theta$ $\approx 0.35$ to $\theta \approx 0.5$, and then drops dramatically to a small value. Eventually larger coverages lead to an exponent $x$ larger than two. Obviously the behavior of $x$ is rather complex and its interpretation is nontrivial. However, based on our discussion and results above, we suggest the following picture.

At very small coverages one finds the system in the disordered phase where the role of ordering is not relevant. Thus the behavior of $x$ at small $\theta$ can be attributed to attractive interactions, which dominate the system in the dilute limit. So, as the attractive adatom-adatom interactions become more significant, they lead to a decrease of $x$ with an increasing coverage. Around $\theta=0.35$ at $T=590 \mathrm{~K}$, however, one enters the $p(2 \times 1)$ phase and consequently the behavior of $x$ is now governed by ordering. In the present case this leads to an increasing $x$ for $0.35 \leqslant \theta \leqslant 0.50$, giving rise to a maximum in $x$ around the ideal coverage of the $p(2 \times 1)$ phase. This maximum relates to enhanced backjump correlations in the adparticle motion, which in the present case are strongest around $\theta=1 / 2 .^{29,39}$ At $\theta=0.50$, the situation changes drastically as diffusion changes from a $2 \mathrm{D}$ to a $1 \mathrm{D}$ process, thus leading to a very small exponent $x$ for 0.50 $<\theta<0.60$. Finally, at large coverages $\theta>0.60$, the role of repulsive interactions becomes more and more important and leads to an increasing exponent which eventually becomes larger than 2 .

This view relies partly on our previous conclusions regarding the temperature dependence, but it is also supported by our interaction results shown in Fig. 2. Although the understanding of the intermediate-coverage behavior is still not complete, we can conclude that the intermediate decay rate of $\widetilde{\phi}_{g}(t)$ depends very strongly on the ordering of the system. In the disordered phase, ordering plays no significant role and then the nature of interactions seems to be a sufficient criterion to determine the region of $x$ : For repulsive interactions we find $x \geqslant 2$, while attractive interactions lead to $x<2$. In ordered phases, however, the same idea is not sufficient for a proper description of $x$. Namely, if one considers the case at high coverage and at low temperature, then the system is dominated by repulsive interactions but still we find $x<2$ within the ordered phases and in the vicinity of phase boundaries. Therefore, within the ordered phases, our present results are in favor of an idea that the behavior of $x$ is governed by ordering effects that determine the local structure in which adatoms diffuse, while the nature of interactions is less important. This idea is supported by additional studies within a lattice-gas model which includes repulsive nearest-neighbor interactions only. This model corresponds to an antiferromagnetic Ising model and is known to have a disordered phase at high temperatures, and an ordered $c(2$ $\times 2$ ) phase at low $T$. Using this model, we have found $x$ $>2$ above the order-disorder transition temperature and $x$ $<2$ at intermediate coverages at low temperatures within the ordered $c(2 \times 2)$ phase, ${ }^{36}$ in agreement with the interpretation above.

\section{B. Results for the model polymer system}

All the results for $\phi_{g}(t)$ shown here have been reported in a previous publication. ${ }^{20}$ For the sake of completeness, however, we summarize these results very briefly. They are in complete qualitative agreement with the results for the $\mathrm{O} / \mathrm{W}(110)$ system.

In the numerical calculations, we used $\tau_{0}=10 \mathrm{MCS}$. In all cases studied, we again found a power-law decay $\phi_{g}(t)$ $\propto t^{-x}$ at intermediate times, with an exponent $x$ whose value depends on the interaction strength. At a fixed coverage $\theta$ $=0.25$, we found $x=2.0 \pm 0.1$ in the athermal limit, $x=1.7$ \pm 0.2 for a weak attractive interaction between the segments of different chains, and $x=1.5 \pm 0.2$ for strongly attractive chains. Since there are no ordered phases under present circumstances, we can conclude that the effective value of $x$ decreases when attractive interactions become stronger.

We also studied how $\phi(t)$ depends on $\theta$. To this end, we used the model without direct interactions, in which case the entropic interchain repulsion increases with coverage. ${ }^{33} \mathrm{We}$ found exponents $x=2.0 \pm 0.1,2.6 \pm 0.2$, and $2.6 \pm 0.4$ for $\theta$ $=0.25,0.5$, and 0.75 in respective order. These results indicate that the effective $x$ may be larger than two with increasing repulsion.

\section{RESULTS FOR VELOCITY CORRELATIONS IN COLLECTIVE DIFFUSION}

\section{A. Results for the model system O/W(110)}

We next considered the decay of velocity correlations in collective diffusion by calculating $\psi_{g}(t)$ numerically with a time step of $\tau_{0}=1 \mathrm{MCS}$. Instead of considering a wide region of parameter space, we decided to focus on the case $\theta$ $=0.55$ and to determine $\psi_{g}(t)$ as accurately as possible at a few temperatures only. This choice is due to the fact that, unlike $\phi_{g}(t), \psi_{g}(t)$ is a collective quantity and therefore its accurate determination requires extensive computational efforts. Furthermore the memory effects in collective diffusion are much weaker than those in tracer diffusion, ${ }^{14}$ which means that $\int_{0}^{t} \psi_{g}\left(t^{\prime}\right) d t^{\prime}$ converges much faster than 


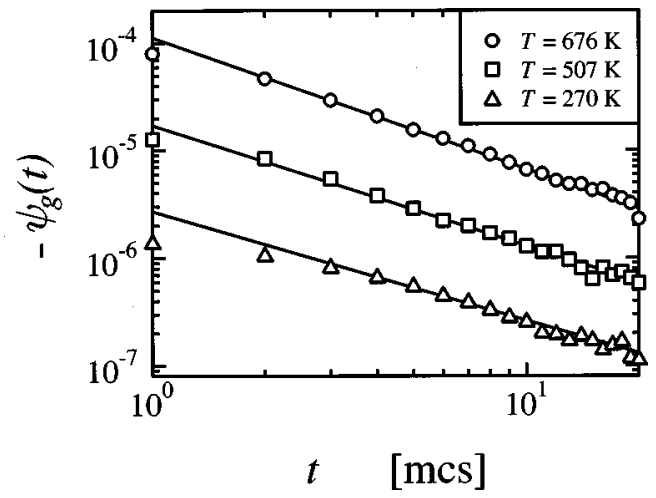

FIG. 7. Typical results for the decay of $\psi_{g}(t) \sim t^{-y}$ at three temperatures at $\theta=0.55$ in the $\mathrm{O} / \mathrm{W}(110)$ model system. Full lines are fits to the data and correspond to exponents $y=1.23(T=676 \mathrm{~K}), y=1.12(T=507 \mathrm{~K})$, and $y=1.02(T=270 \mathrm{~K})$. The lowest curve has been shifted for clarity.

$\int_{0}^{t} \phi_{g}\left(t^{\prime}\right) d t^{\prime}$. This implies that the time window in which a power-law decay is expected to hold, is considerably narrower in case of $\psi_{g}(t)$.

The correlation function $\psi_{g}(t)$ was found to have a positive leading term $\psi_{g}(0)$ and a negative tail at times $t>0$. Typical results for $\psi_{g}(t)$ are shown in Fig. 7. We find that after a very small crossover time, an effective power-law form $\psi_{g}(t) \propto t^{-y}$ develops at intermediate times. The exponent $y$ was found to decrease monotonically as the temperature is lowered from high to low temperatures: we found $y$ $=1.23 \pm 0.05$ at $T=676 \mathrm{~K}$ and $y=1.02 \pm 0.10$ at $T=270 \mathrm{~K}$. Therefore, to make the story brief, we conclude that the results for $\psi_{g}(t)$ are in qualitative agreement with previous results for $\psi_{g}(t)$ and suggest that $y$ is nonuniversal and thus subject to interaction effects.

\section{B. Results for the model polymer system}

To complement previous studies, we carried out additional simulations for the model polymer system. We first considered the model without direct interactions and varied the coverage $\theta$. As shown in Fig. 8, there is an approximate power-law decay $\psi_{g}(t) \sim t^{-y}$ at relatively short times. The time window in which the power-law decay is observed is very narrow, since the memory effects in collective diffusion

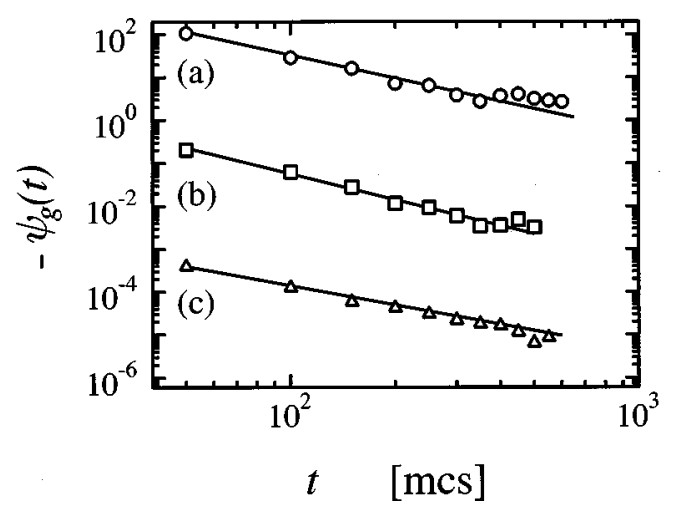

FIG. 8. Results for the decay of $\psi_{g}(t) \sim t^{-y}$ in the model polymer system. Results are shown for the cases (a) $\theta=0.40$ and (b) $\theta=0.65$ without direct interactions, and (c) at $\theta=0.40$ for strongly attractive chains. are very weak. ${ }^{14}$ This, again, implies that the computational effort in studies of $\psi_{g}(t)$ is extensive, and makes an accurate determination of $y$ very difficult. In the present case, we found exponents $y=1.8 \pm 0.1$ at $\theta=0.40$ and $y=2.0 \pm 0.1$ at $\theta=0.65$. This behavior is in agreement with results for the decay of $\phi_{g}(t)$. Additionally, we studied the decay of $\psi_{g}(t)$ for strongly attractive chains and found $y=1.5 \pm 0.1$ at $\theta$ $=0.40$, again in agreement with the behavior of $\phi_{g}(t)$. These results confirm that the decay of $\psi_{g}(t)$ follows, qualitatively, the same behavior as the decay of velocity correlations in tracer diffusion.

\section{DISCUSSION}

It is widely accepted that in various surface systems adatoms move by a sequence of jumps from one adsorption site to another. In these cases, one often assumes that each jump is thermally activated and characterized by a well-defined activation barrier. Consecutive jumps by the adatom, in turn, are often assumed to be uncorrelated events just like in a random walk. Due to its simplicity, this idea is often used to analyze experimental data in the context of Arrhenius description, thus yielding some activation barrier for the diffusion process. Despite its common use, this approach is problematic for two reasons. First, recent studies have demonstrated that interactions with other adatoms affect the potential energy surface felt by the tagged adatom. ${ }^{37,38}$ Therefore even in the low-coverage limit, ${ }^{38}$ the diffusion barrier extracted from an Arrhenius analysis is some complex average of various activation barriers rather than a welldefined activation energy of some individual process. Second, interactions with neighboring adatoms also influence the motion of the tagged adatom, leading to a fact that the adatom executes correlated jumps from one adsorption site to another. In the present work as well as in recent works, we have demonstrated ${ }^{14,23,29,39}$ that these correlations (memory effects) can become very important for strongly interacting systems, and may even change the effective Arrhenius barriers significantly. ${ }^{23,39}$

In this paper, we have considered such memory effects on a very general level, namely, in terms of velocity correlations which actually give rise to the diffusion coefficients in the first place. We have first generalized the conventional definition of velocity autocorrelation functions to those corresponding to discrete time steps. These generalized correlation functions, namely, $\phi_{g}(t)$ for tracer diffusion and $\psi_{g}(t)$ for collective diffusion, are defined in terms of adatom displacements during a given time interval, and can therefore be directly related to experimental measurements. Then, we have demonstrated through the study of two strongly interacting model systems how the memory effects manifest themselves in a nontrivial temporal behavior of $\phi_{g}(t)$ and $\psi_{g}(t)$. In cases where the interparticle interactions are important, we find that both $\phi_{g}(t)$ and $\psi_{g}(t)$ display a transient nonexponential regime at intermediate times, well approximated by algebraic decays of the form $\phi_{g}(t) \sim t^{-x}$ and $\psi_{g}(t) \sim t^{-y}$. This behavior is most pronounced in $\phi_{g}(t)$ for tracer diffusion, where the memory effects seem to be much stronger than in collective diffusion. Based on our studies, the decay exponent $x$ is approximately two in totally disor- 
dered phases as characterized by the Langmuir gas model, while predominantly attractive (repulsive) adatom-adatom interactions within the disordered phase tend to decrease (increase) this exponent. In ordered phases, the case is different. Then we found that the behavior of $x$ depends very much on the ordering of the system, leading to $1<x<2$ in phases where diffusion is truly two-dimensional. Under circumstances where diffusion of adatoms is one-dimensional, however, we found strong evidence for a regime that is characterized by a very small exponent $x<1$. The conclusion that can be drawn from the present work is that, within a disordered phase, the behavior of $x$ is related to the nature of predominant interactions, while in ordered phases $x$ is mainly governed by the local ordering of the adlayer. This should be particularly useful from the experimental point of view to obtain information about the ordering and the nature of predominant interactions between the adparticles.

The results presented here seem to indicate that a nonexponential intermediate time dependence of the velocity correlation functions is a general feature in dissipative 2D adsorption systems, and arises from the backcorrelation effect in the absence of hydrodynamic interactions and conservation laws. We note that in such systems, there is an additional source of memory effects through the coupling to the underlying substrate excitations which has been so far studied only in the case of individual adatoms. ${ }^{16}$ Such memory effects are likely to play a role at times that are comparable to the vibrational motion of adparticles, and are therefore not accounted for in the present work. It is interesting to note that when hydrodynamic interactions are present, intermediate power-law decay of $\phi(t)$ has also been observed in dissipative $2 \mathrm{D}$ hard sphere fluids, ${ }^{21}$ and in diffusive particle dynamics under steady-state sedimentation conditions. ${ }^{25}$ It would be of theoretical interest to understand these effects through further analytic work. The memory effects could also be measured directly by experimental techniques such as STM. Admittedly, the requirement of high coverage for sizable memory effects would make the task of identifying and following specific adparticles difficult in a STM experiment. The most favorable condition would be for systems where there are long-range interactions such as dipoledipole interactions between adsorbed alkali metals. For these systems, the memory effect should manifest itself at relatively low coverages.

\section{ACKNOWLEDGMENTS}

This work has in part been supported by the Academy of Finland through its Center of Excellence Program and by a grant from the European Union (I.V.).

\footnotetext{
${ }^{1}$ A.-L. Barabási and H. E. Stanley, Fractal Concepts in Surface Growth (Cambridge University Press, Cambridge, 1995).

${ }^{2}$ V. P. Zhdanov and B. Kasemo, Surf. Sci. Rep. 20, 111 (1994).

${ }^{3}$ R. Gomer, Rep. Prog. Phys. 53, 917 (1990).

${ }^{4}$ J. P. Boon and S. Yip, Molecular Hydrodynamics (Dover, New York, 1980).

${ }^{5}$ T. Ala-Nissila and S. C. Ying, Prog. Surf. Sci. 39, 227 (1992)

${ }^{6}$ A. Danani, R. Ferrando, E. Scalas, and M. Torri, Int. J. Mod. Phys. B 11, 2217 (1997).
}

${ }^{7}$ A. G. Naumovets and Yu. S. Vedula, Surf. Sci. Rep. 4, 365 (1985).

${ }^{8}$ G. L. Kellogg, Surf. Sci. Rep. 21, 1 (1994), and references therein.

${ }^{9}$ T. R. Linderoth, S. Horch, L. Petersen, S. Helveg, E. Laegsgaard, I. Stensgaard, and F. Besenbacher, Phys. Rev. Lett. 82, 1494 (1999); F. Montalenti and R. Ferrando, ibid. 82, 1498 (1999), and references therein.

${ }^{10}$ J. Merikoski, I. Vattulainen, J. Heinonen, and T. Ala-Nissila, Surf. Sci. 387, 167 (1997), and reference therein.

${ }^{11}$ K. A. Fichthorn, Adsorption 2, 77 (1996).

${ }^{12}$ L. J. Lauhon and W. Ho, J. Chem. Phys. 111, 5633 (1999).

${ }^{13}$ T. Hjelt, I. Vattulainen, J. Merikoski, T. Ala-Nissila, and S. C. Ying, Surf. Sci. 380, L501 (1997); 402-404, 253 (1998).

${ }^{14}$ S. C. Ying, I. Vattulainen, J. Merikoski, T. Hjelt, and T. Ala-Nissila, Phys. Rev. B 58, 2170 (1998).

${ }^{15}$ L. Y. Chen and S. C. Ying, Phys. Rev. B 49, 13838 (1994); L. Y. Chen, M. R. Baldan, and S. C. Ying, ibid. 54, 8856 (1996).

${ }^{16}$ A. Cucchetti and S. C. Ying, Phys. Rev. B 54, 3300 (1996).

${ }^{17}$ R. Ferrando, R. Spadacini, G. E. Tommei, and G. Caratti, Surf. Sci. 311, 411 (1994); F. Montalenti and R. Ferrando, Phys. Rev. B 59, 5881 (1999).

${ }^{18}$ B. J. Alder and T. E. Wainwright, Phys. Rev. A 1, 18 (1970); D. Levesque and W. T. Ashurst, Phys. Rev. Lett. 33, 277 (1974).

${ }^{19}$ C. P. Lowe and D. Frenkel, Physica A 220, 251 (1995); see also M. H. J. Hagen, I. Pagonabarraga, C. P. Lowe, and D. Frenkel, Phys. Rev. Lett. 78, 3785 (1997); I. Pagonabarraga, M. H. J. Hagen, C. P. Lowe, and D. Frenkel, Phys. Rev. E 58, 7288 (1998); I. Pagonabarraga, M. H. J. Hagen, C. P. Lowe, and D. Frenkel, ibid. 59, 4458 (1999).

${ }^{20}$ T. Hjelt, I. Vattulainen, T. Ala-Nissila, and S. C. Ying, Surf. Sci. 449, L255 (2000)

${ }^{21}$ P. B. Sunil Kumar and M. Rao, Phys. Rev. Lett. 77, 1067 (1996); C. P. Lowe, R. van Roij, and D. Frenkel, ibid. 79, 1168 (1997); P. B. Sunil Kumar and M. Rao, ibid. 79, 1169 (1997).

${ }^{22}$ One possible way to measure $\phi(t)$ in terms of particle displacement information is the scanning tunneling microscopy; see B. S. Swartzentruber, Phys. Rev. Lett. 76, 459 (1996); Surf. Sci. 374, 277 (1997).

${ }^{23}$ I. Vattulainen, S. C. Ying, T. Ala-Nissila, and J. Merikoski, Phys. Rev. B 59, 7697 (1999).

${ }^{24}$ N. H. March and M. P. Tosi, Atomic Dynamics in Liquids (Dover, New York, 1976).

${ }^{25}$ E. Kuusela and T. Ala-Nissila, Phys. Rev. E (submitted).

${ }^{26}$ G.-C. Wang, T.-M. Lu, and M. G. Lagally, J. Chem. Phys. 69, 479 (1978).

${ }^{27}$ C. R. Brundle and J. Q. Broughton, The Chemical Physics of Solid Surfaces and Heterogeneous Catalysis: Chemisorption Systems, edited by D. A. King and D. P. Woodruff (Elsevier, Amsterdam, 1990), Vol. 3A, Chap. 3.

${ }^{28}$ K. E. Johnson, R. J. Wilson, and S. Chiang, Phys. Rev. Lett. 71, 1055 (1993).

${ }^{29}$ I. Vattulainen, J. Merikoski, T. Ala-Nissila, and S. C. Ying, Phys. Rev. B 57, 1896 (1998).

${ }^{30}$ I. Vattulainen, S. C. Ying, T. Ala-Nissila, and J. Merikoski, J. Chem. Phys. 111, 11232 (1999); I. Vattulainen, J. Merikoski, T. Ala-Nissila, and S. C. Ying, Phys. Rev. Lett. 80, 5456 (1998).

${ }^{31}$ I. Carmesin and K. Kremer, Macromolecules 21, 2819 (1988); Monte Carlo and Molecular Dynamics Simulations in Polymer Science, edited by K. Binder (Oxford, New York, 1995).

${ }^{32}$ Here, one MCS is defined as an attempt to move each monomer of every chain in the model.

${ }^{33}$ T. Ala-Nissila, S. Herminghaus, T. Hjelt, and P. Leiderer, Phys. Rev. Lett. 76, 4003 (1996); T. Hjelt, S. Herminghaus, T. Ala-Nissila, and S. C. Ying, Phys. Rev. E 57, 1864 (1998)

${ }^{34}$ D. K. Chaturvedi, Phys. Rev. B 28, 6868 (1983); J. Phys. C 17, L449 (1984).

${ }^{35}$ We note that at low coverages within the Langmuir gas model, the powerlaw decay of $\phi(t)$ is not pronounced due to the weakness of memory effects in this case. For the same reason for any system at a low coverage, we expect a relatively narrow time window in which $\phi(t)$ decays like a power-law. Studies of $\phi(t)$ at low $\theta$ are obviously rendered by this problem.

${ }^{36}$ I. Vattulainen et al. (unpublished).

${ }^{37}$ S. Renisch, R. Schuster, J. Wintterlin, and G. Ertl, Phys. Rev. Lett. 82, 3839 (1999).

${ }^{38}$ M. Ø. Pedersen, L. Österlund, J. J. Mortensen et al., Phys. Rev. Lett. 84, 4898 (2000).

${ }^{39}$ I. Vattulainen, Surf. Sci. 412-413, L911 (1998). 